\title{
Ecological Strategies and Knowledge Mapping
}

\author{
Jose Bidarra $^{1}$ and Ana Dias ${ }^{2}$ \\ ${ }^{1}$ Universidade Aberta, \\ Rua da Escola Politecnica 147, \\ 1269-001 Lisboa, Portugal \\ bidarra@univ-ab.pt \\ ${ }^{2}$ TecMinho, \\ Universidade do Minho, \\ Campus de Azurem, \\ 4800-058 Guimaraes, Portugal \\ anadias@tecminho.uminho.pt
}

\begin{abstract}
In this paper we discuss the concept of ecological strategy in relation to cognitive artefacts that reflect the expressive power of learners engaged in the development of significant knowledge paths. Our experiments show that learners strive to make sense of the fragmentary information surrounding them and in this endeavour they may benefit from the creation of knowledge maps, i.e. branched structures of knowledge that depart from a central node, a starting point or a homepage. In the course of creating these fractal hyperspaces on the Web, with integration of varied multimedia materials, the range of cognitive strategies used during the learning process becomes evident, both at individual and collective level. Perhaps ecological strategies may encourage a new research approach regarding the study of knowledge construction within complex hypermedia environments.
\end{abstract}

\section{Introduction}

Inspired by Resnick's eThinking [5], we discuss in this paper the need for a conceptual framework integrating multimedia hyperscapes [1], [4], with a new way of thinking and a cultural perspective that go beyond the traditional "clockwork approach" to reach a further "ecological approach". Ever since the $17^{\text {th }}$ Century we have been presented with Newton's mechanical view of the universe, nonetheless, in the present day new ideas from ecology, ethology and evolution are influencing scientific research in many areas. Ecological strategies are very common in the biological world and share two common characteristics: response to local conditions and adaptation to change. These can also be described as decentralized, simple, flexible and robust problem solving strategies that transfer without difficulty to networked environments.

A network is, in its essence, a tissue of collaborations of many parts that can create a critical mass and reach certain states in an extremely effective way. Taking the WWW network, covering the whole planet, we can say that its complexity is so big that at the local level we do not perceive the global changes that are taking place. We just scratch on the surface and this has consequences for our lives. Perhaps we are at 
the threshold of a new culture, no doubt very rich in information, that forces us to read history in a non-linear way. In other words, sequential reasoning becomes difficult as we try to catch up and order the many fragments of information that reach us every hour, every minute, every second.

In this Web ecosystem we are becoming - perhaps our "selves" are becoming digital like in character. Our identities, our communications, our work is being constructed by and through digital technology (e-mail, homepages, courseware, etc.). Any division between people and technology is becoming more and more artificial as we are constantly building hypermedia spaces that grow in an organic and generative way in the course of human interactions. This is the environment where learning occurs these days. For instance, when we have a large number of students connected through the network, we can create activities based on ecological principles, since these involve the participation of all the contributors and the combination of the best solutions to solve complex problems. Also, the development of new tools, software or digital artifacts, stimulates the creation of communities of practice (developers, experts, students, teachers), engaged in a construction process.

\section{Ecological Learning Strategies}

The creation of multimedia hyperscapes [1], [4], in essence the construction of networked or branching cognitive artefacts, tries to reflect the expressive power of learners in the development of significant knowledge paths. As people try to make sense of the fragmentary information surrounding them they tend to create branched structures of knowledge that depart from a single node, a starting point or a homepage. In the beginning there is something that triggers new thoughts, perhaps an unexpected question or a provoking idea.

In our experiments at Universidade Aberta the construction of knowledge is achieved through concept mapping and related cognitive activities. Since the Web makes possible new modes of collaboration and interaction, groups of students can work together very effectively on such tasks as gathering information, designing Web pages, discussing relevant topics, organizing events, writing assignments, making multimedia presentations, among other. In the course of creating fractal hyperspaces on the Web, with integration of varied multimedia materials, the range of cognitive strategies used by students during the learning process becomes evident, both at individual and collective level. As a result, assessment of portfolios turns out a straightforward task for the professor or trainer.

But reality is somehow more complex. Constructing knowledge by means of multimedia hyperscapes represents a shift from a very centralized didactic style, based on the teacher as the main source of knowledge, to a flexible networked learning environment where the student must be able to construct meaningful structures through connections to multiple sources of information. In particular, it implies ecological habits of thinking within a vast, complex and unpredictable environment. Immersed in a technological maze - the World Wide Web is often confusing - new modes of interaction seem to materialize. After studying the modes of hypermedia development used by students and teachers working as a team, relying on cognitive and concept mapping tools, we may understand the (varied) mechanisms used by learners to dominate the complexity of the vast available resources. 
Furthermore, the Web may also be compared to natural ecosystems because it has no centralized decision-making device. Paradoxically, there is no apparent leadership to tell learners what to do but the ecologies of the Web make possible the use of information and knowledge to attain effective global results.

In this context is important to distinguish between knowledge and information; according to Salomon [6]:

- Information is discrete, knowledge is arranged in networks with meaningful connections between the nodes.

- Information can be transmitted as is; knowledge needs to be constructed as a web of meaningful connections.

- Information need not be contextualized; knowledge is always part of a context.

- Information requires clarity; the construction of knowledge is facilitated by ambiguity, conflict and uncertainty.

- Mastery of information can be demonstrated by its re-production; mastery of knowledge is demonstrated by its novel applications. (p. 4)

Eventually a greater problem emerges: how do we integrate interaction processes, authoring approaches and rich elements of hypermedia information in order to improve the effectiveness of educational environments? Are ecological strategies the answer to the mounting complexity of current learning systems?

\section{Designing Knowledge Hyperscapes}

Ecological learning strategies may be supported by the construction of multimedia hyperscapes; in this regard we must consider that Web design activities:

- introduce the notion of hyperlinked knowledge spaces enriched with digital multimedia (i. e. establishes the concept of a complex ecosystem);

- suggest a potential for the development of highly interactive constructivist learning environments (i. e. enables learner's response to given situations);

- permit the creation and growth of networked learning communities using synchronous and asynchronous communication devices (i. e. facilitates adaptation to new or changing conditions).

Within our learning activities at Universidade Aberta we are developing models that enable the construction of hyperscapes through knowledge mapping. This technique was developed for representing knowledge in graphs that constitute treelike structures [2], [3]. These consist of nodes and links, where nodes represent concepts and links represent the relations between concepts. Concepts, and sometimes links, are labeled and may be categorized. The developing patterns of association and branching create fractal structures. Like clouds or trees, they form physical structures that do not possess a defined form; yet in fractal structures we can always describe other levels or scales of its structure where we always find the same basic elements (self-similarity). Fractal geometry establishes algorithms to describe/create fractal structures but these are not relevant at this time. Knowledge maps or hyperscapes may have the aspect of fractal structures (Fig. 1). 


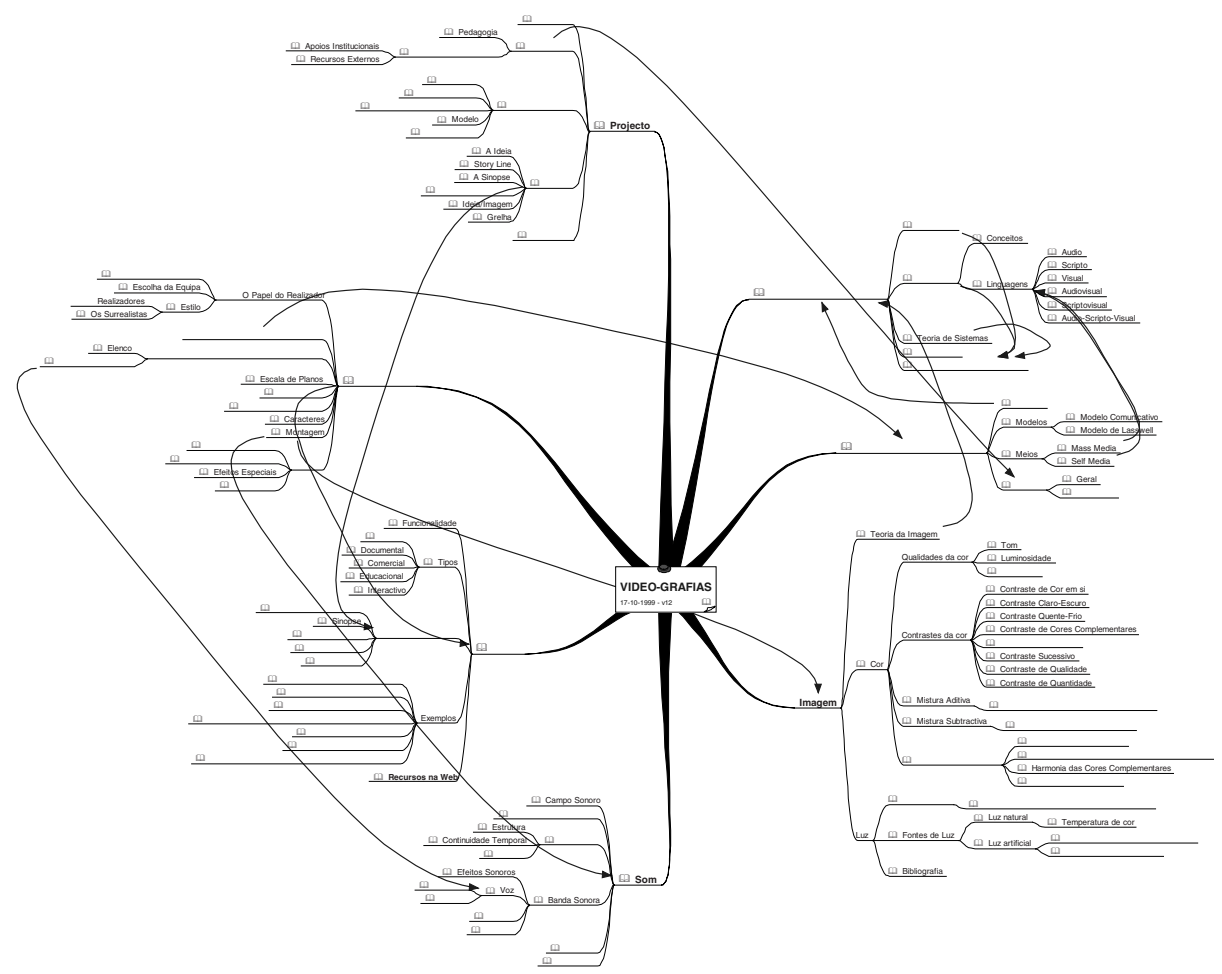

Fig. 1. Global visualization of a branched knowledge structure created with a mapping tool (MindManager ${ }^{\mathrm{TM}}$ ).

While recognizing the value of knowledge mapping, we were able to witness in our experiments that authoring with hypermedia tools is a rather difficult task for most people. Content creation, whatever the form and technology used, is a huge challenge for the average person and this explains why we have professional Web designers, movie makers, writers and musicians. Nevertheless, we believe that future scientists will also be artists - they will go beyond the "exact words" to reverberate across the culture shared with their audience. By establishing the right context we may have more success in recreating each scientist's own experience. The way to establish context, as we propose here, is through ecological thinking and the construction of multimedia hyperscapes.

\section{Future Learning Environments}

We have focused on the previous section on the creation of multimedia hyperscapes departing from an ecological thinking position. Basically, when we have a number of learners connected over the network we can create activities based on ecological principles that involve the evaluation of all the relevant information and the combination of the best solutions to solve complex problems within a suitable time. 
The preliminary findings of our research suggest that activities leading to the mapping of concepts are effective to motivate students and increase the level of interaction with learning resources. We believe that any subject matter will be mastered more readily and more thoroughly when students become able to derive intrinsic rewards from dealing with complex domains. On the other hand, ecological ideas are possible to apply only within a set environment with a specific group of people trying to solve relevant problems, transforming information into knowledge.

Nonetheless, the construction of knowledge is a problem of conceptual development based on an evolutionary process with a rather unpredictable outcome. Implicit in this process are pro-active learning strategies, the collaboration with peers and other students and the adoption of a bold perspective concerning the problems to solve. In this context knowledge representations may take many forms. For instance, "chaotic" elements that enter the processes in creative activities (e.g. generation of ideas) have to be managed ad hoc, according to each learner's path and progression, to arrive at a final conceptual representation that is valid. This does not mean that a less professional approach is applied but that a great deal of the "authority" usually attributed to the teacher is not possible or desirable. This is a problematic notion that certainly points to a clear paradigm change in education.

Every time ecological strategies are chosen, decisions are based on emerging information, not on fixed or planned solutions. As the learner's environment changes, ecological strategies adjust and deliver new solutions adapted to new circumstances. Our challenge now is to find out how hypermedia mapping tools enable students to engage with their subject matter while working in communities of practice and adopting a symbolic world that will continue to provide curiosity and interest.

\section{References}

1. Bidarra, J., N. Guimarães, P. Kommers (2001). Handling Hypermedia Complexity: Fractal Hyperscapes and Mind Mapping. CINTEC 2001, Aveiro.

2. Buzan, T. (1995). The MindMap Book (2 ed.), BBC Books, London, UK.

3. Gaines, B. R., Shaw, M. L. G. (1995). Concept Maps as Hypermedia Components. URL: http://ksi.cpsc.ucalgary.ca/articles/ConceptMaps/CMa.html. Accessed February 1998)

4. Guimarães, N., T. Chambel, J. Bidarra (2000). From Cognitive Maps to Hypervideo: Supporting Flexible and Rich Learner-Centred Environments. Interactive Multimedia Electronic Journal of Computer-Enhanced Learning (Wake Forest University - USA). URL: http://imej.wfu.edu/articles/2000/2/03/index.asp. Accessed October 2000.

5. Resnick, M. (2002). Thinking Like a Tree (and Other Forms of Ecological Thinking). URL: http://llk.media.mit.edu/projects/eThinking/tree.html. Accessed June 2002.

6. Salomon, G. (2000). It's Not Just the Tool, But the Educational Rationale that Counts. Keynote Speech, ED-MEDIA 2000, Montreal. 